Supporting File.

\title{
In situ biomanufacturing of small molecules in the mammalian gut by probiotic Saccharomyces boulardii.
}

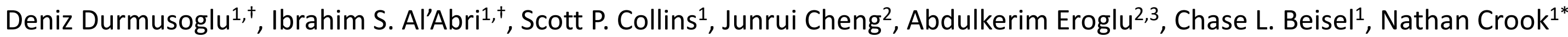

Page 2. Figure S1. Characterization of Fluorescent Genes.

Page 3. Figure S2 Characterization of selective markers and origins of replication.

Page 4. Figure S3 Characterization of Promoters.

Page 5. Figure S4 Comparison of constitutive promoter activity between S. cerevisiae and S. boulardii.

Page 6. Figure S5 Characterization of Terminators.

Page 7. Figure S6. Colony PCR of $S$. boulardii genome editing using only homologous recombination.

Page 8. Figure S7. Genome editing plasmids.

Page 9. Figure S8. Colony PCR of $S$. boulardii genome editing using CRISPR/SpCas9 and CRISPR/LbCas12a.

Page 10. Figure S9 Effect of chromosomal locus and promoter choice on the expression of the fluorescent genes $m R$ Ruby 2 and $m T u r q o u i s e 2$.

Page 11. Figure S10. in vivo Combinatorial Pathway Assembly in S. boulardii.

Page 12. Figure S11. Combinatorial assembly of violacein pathway assembly in S. boulardii.

Page 13. Figure S12. Dosage impacts the residence time of $S$. boulardii in germfree mice.

Page 14. Figure S13. S. boulardii Minimum Inhibitory Concentration (MIC) Tests.

Page 15. Table S1. MoClo kit promoters blasted against S.boulardii's genome

Page 16. Table S2. Integration locations (INT1-INT5) in S. boulardii's genome.

Page 17. Table S3. Guide RNA sequences used for genome editing assisted with the CRISPR nucleases SpCas9 or LbCas12a.

Page 18. Table S4. S. boulardii genome editing efficiencies using 3 different methods.

Page 19. Supplementary text: Beta-carotene production rate calculation. 


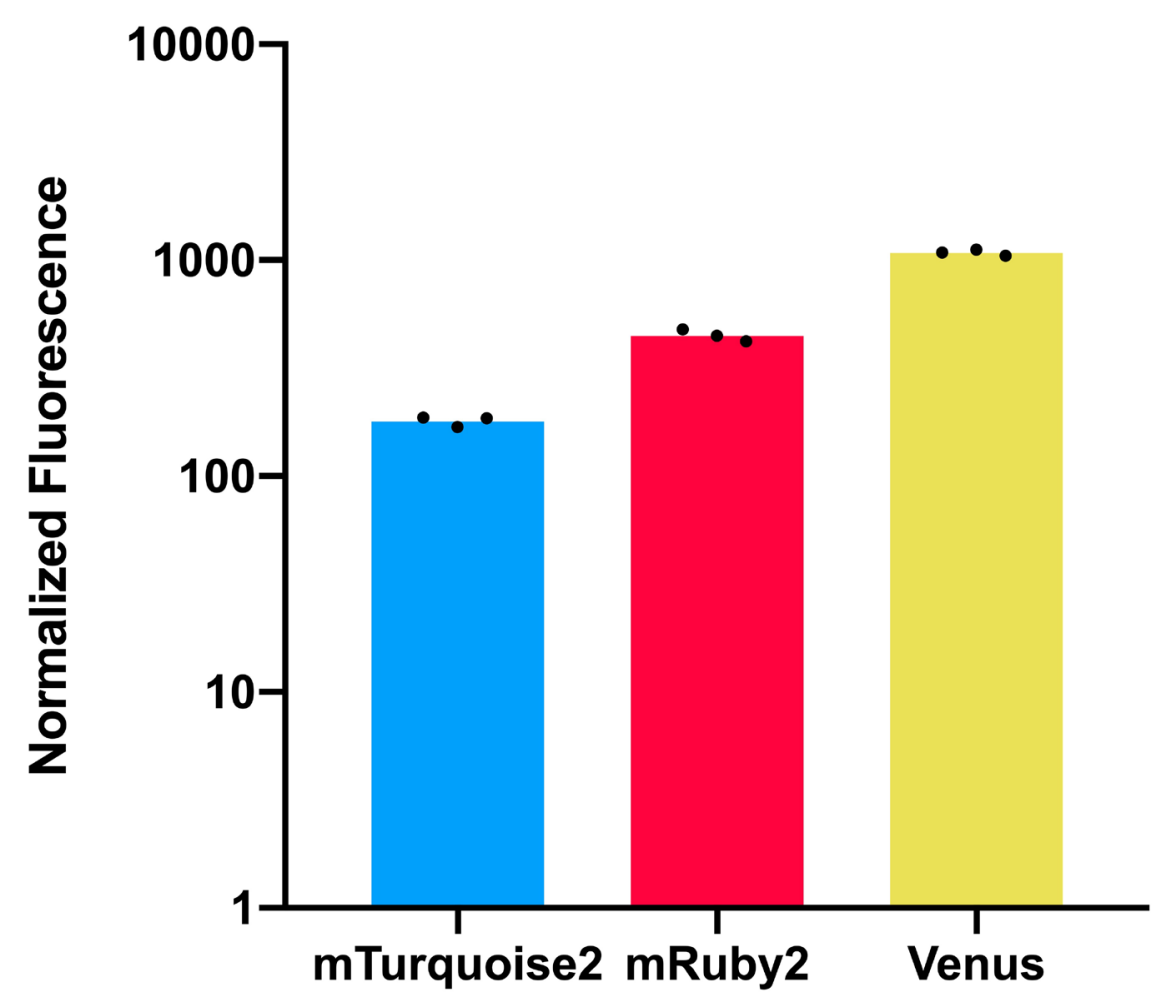

b

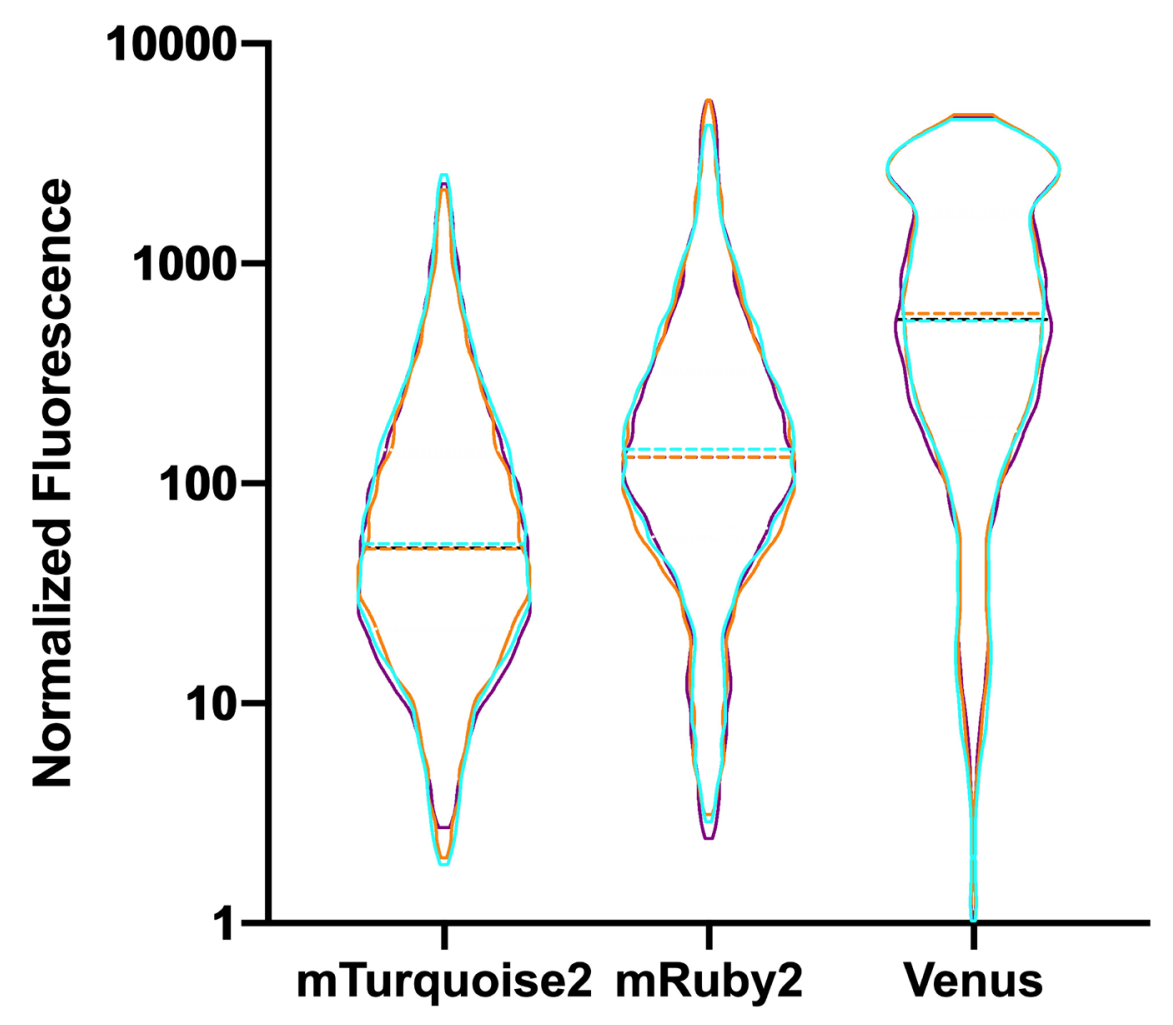

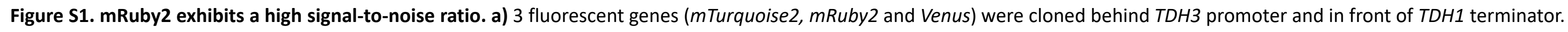

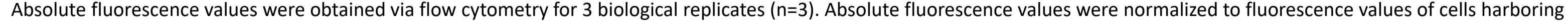

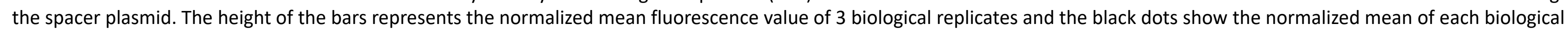

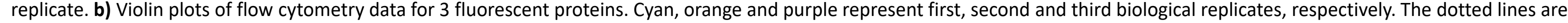
medians of normalized fluorescence for each replicate. Each flow cytometry run consists of 10000 events. 


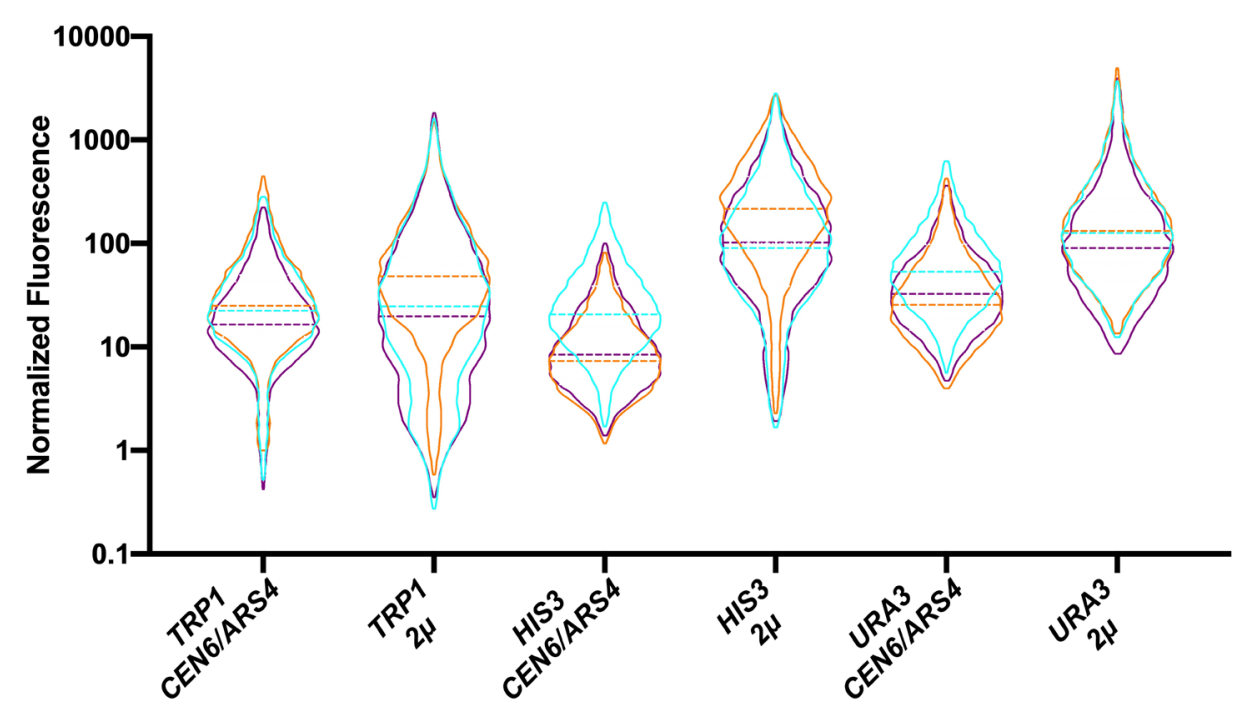

b

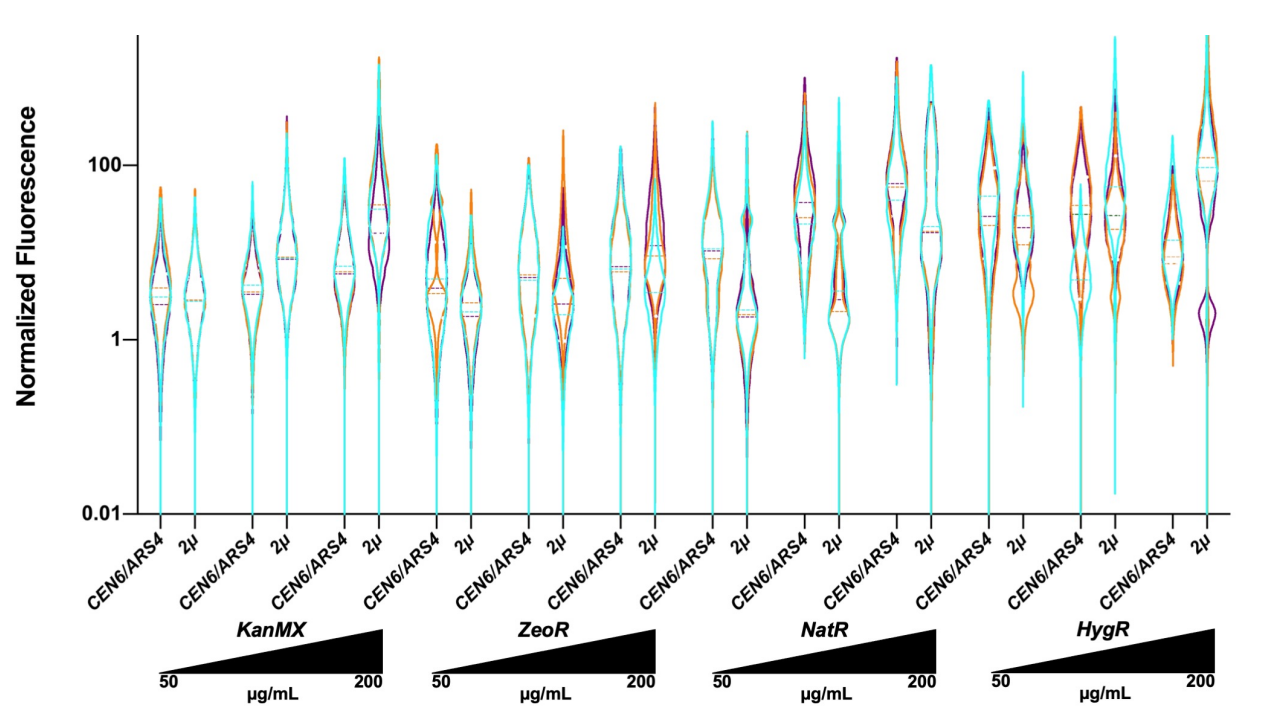

C

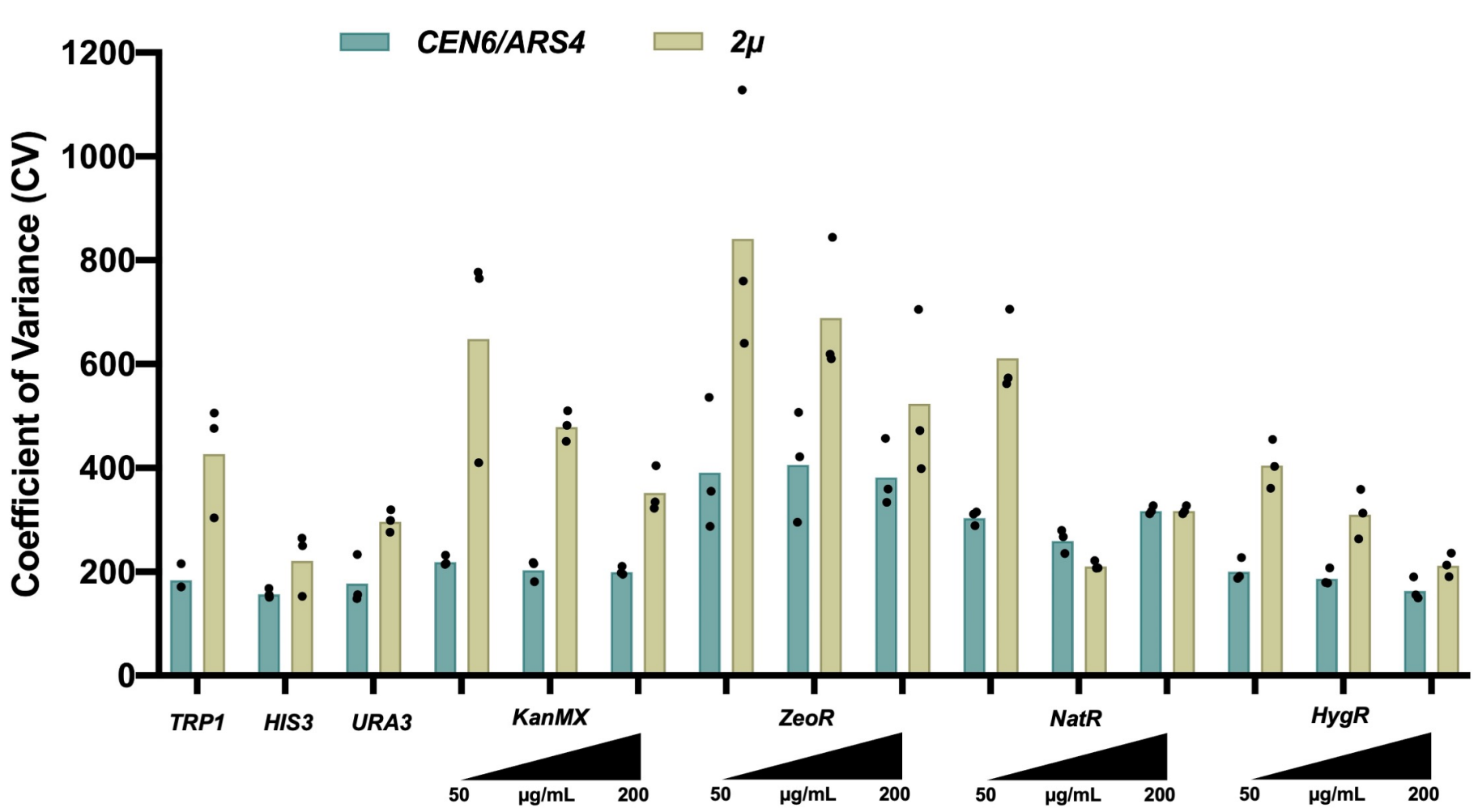

Figure S2. Population-scale variation in plasmidic gene expression enabled by selective markers and origins of replication in S. boulardii. a) Violin plots of flow cytometry data for 3 auxotrophic markers and 2 origins of replication. b) Violin plots of flow cytometry data for 4 antifungal markers and 2 origins of replication tested in 3 different antifungal concentrations (50, $100,200 \mu \mathrm{g} / \mathrm{mL}$ ). 3 auxotrophic markers, 4 antifungal markers and 2 origins of replications were cloned behind TDH3 promoter, mRuby 2 fluorescent protein and TDH1 terminator. Cyan, orange and purple represent first, second and third biological replicates, respectively. The dotted lines are medians of normalized fluorescence for each replicate. Each flow cytometry run consists of 5000-10000 events. c) Coefficient of variance (CV) for 3 auxotrophic markers, 7 antifungal markers tested in 3 different antifungal concentrations (50, 100, 200 $\mu \mathrm{g} / \mathrm{mL}$ ) and 2 origins of replication. The height of the bars represents the mean $\mathrm{CV}$ of 3 biological replicates and the black dots show the $\mathrm{CV}$ of each biological replicate. 


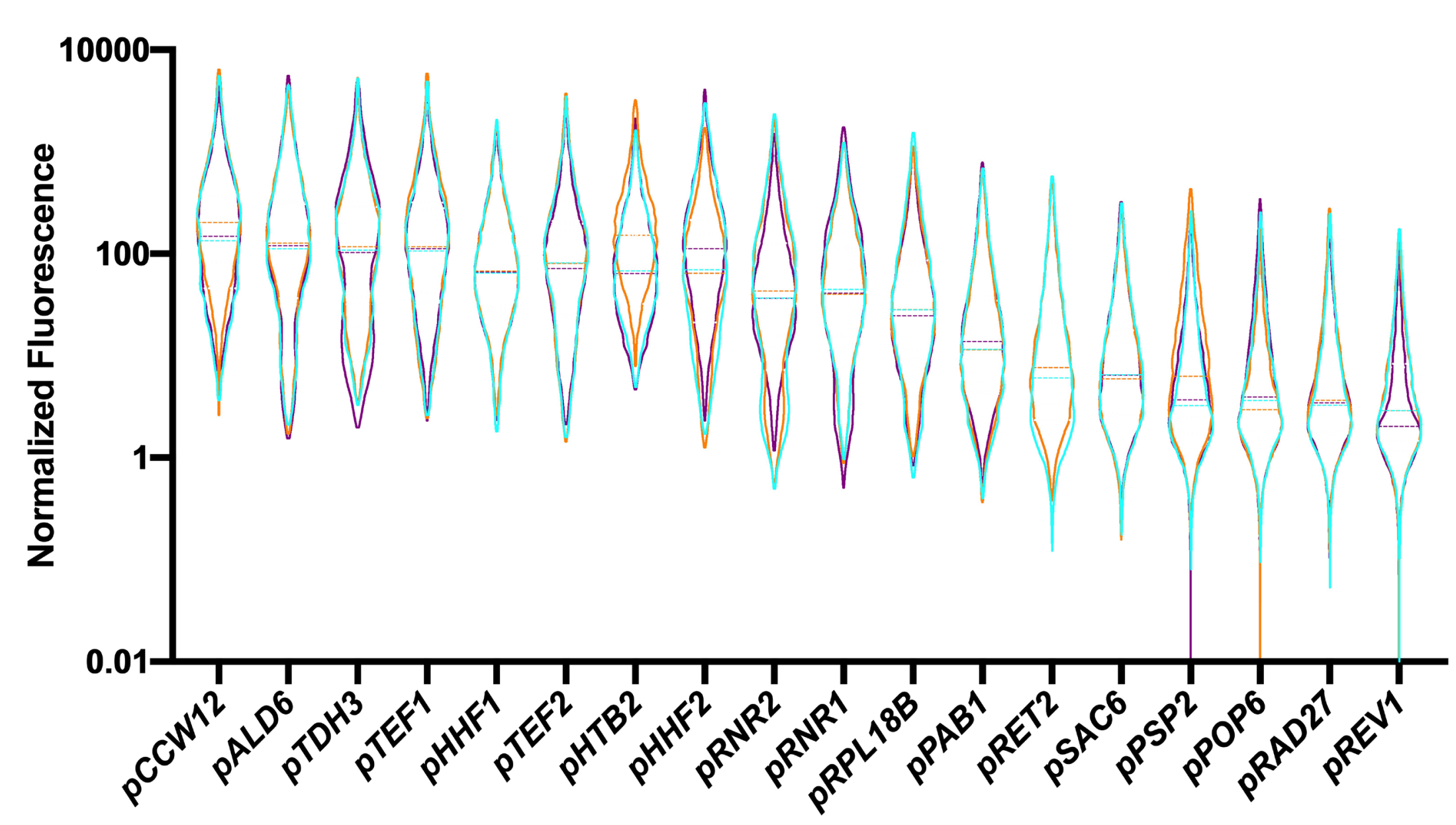

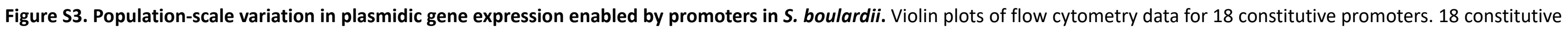

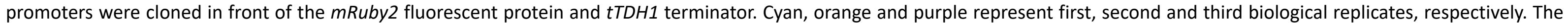
dotted lines are medians of normalized fluorescence for each replicate. Each flow cytometry run consists of 10000 events. 


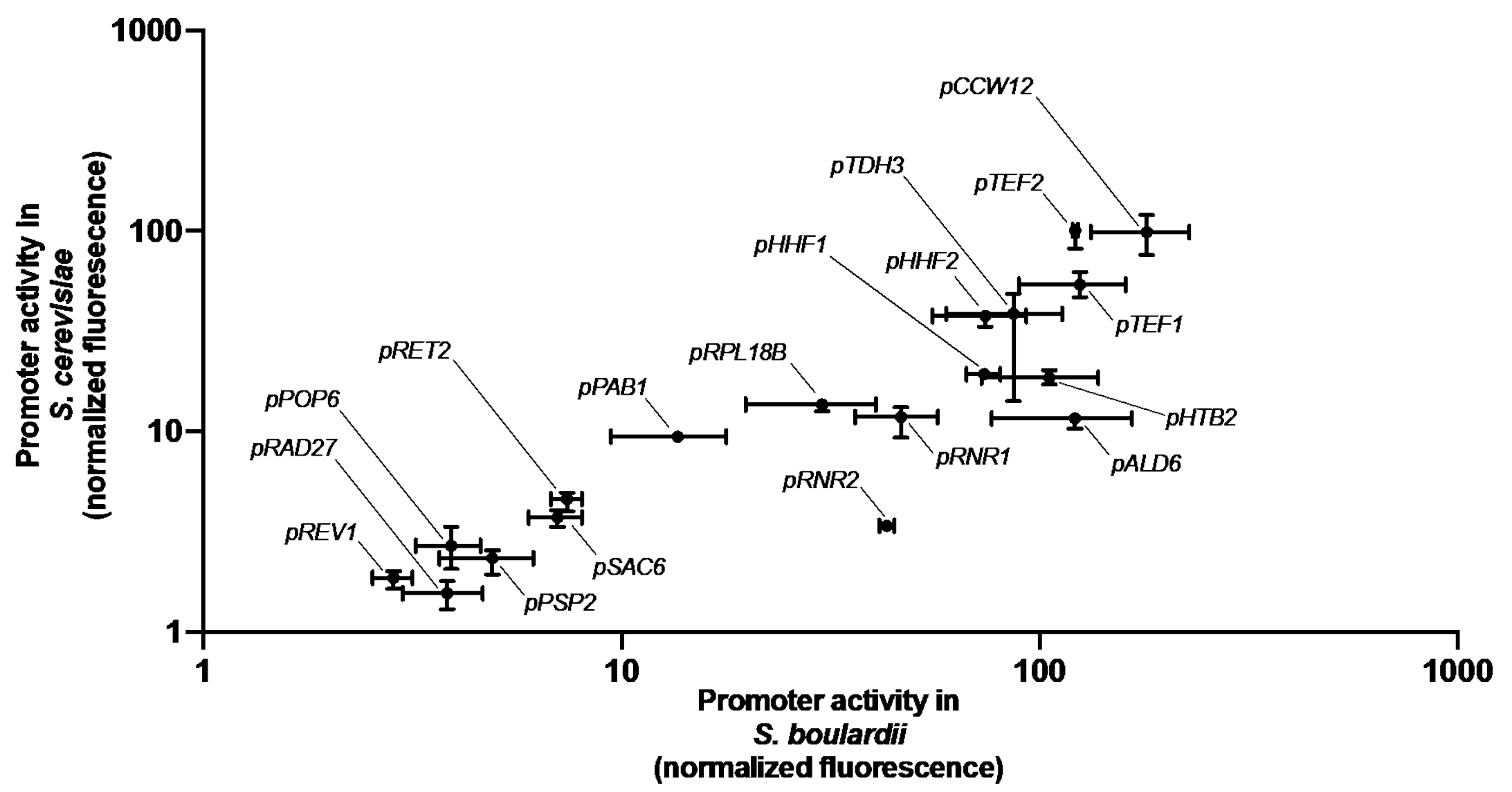

Figure S4. Comparison of constitutive promoter activity between $S$. cerevisiae and S. boulardii. Fluorescence enabled by 18 constitutive promoters in $S b$ ( $x$-axis) and $S$. cerevisiae ( $y$-axis) were plotted $(\mathrm{R}=0.81)$. In each synthetic construct, one constitutive promoter was cloned upstream of $m R$ Ruby 2 gene. Dots correspond to the average medians of 3 (Sb) and 4 ( $S$. cerevisiae) biological replicate populations, and the bars correspond to maximum and minimum median value obtained by the replicates. 


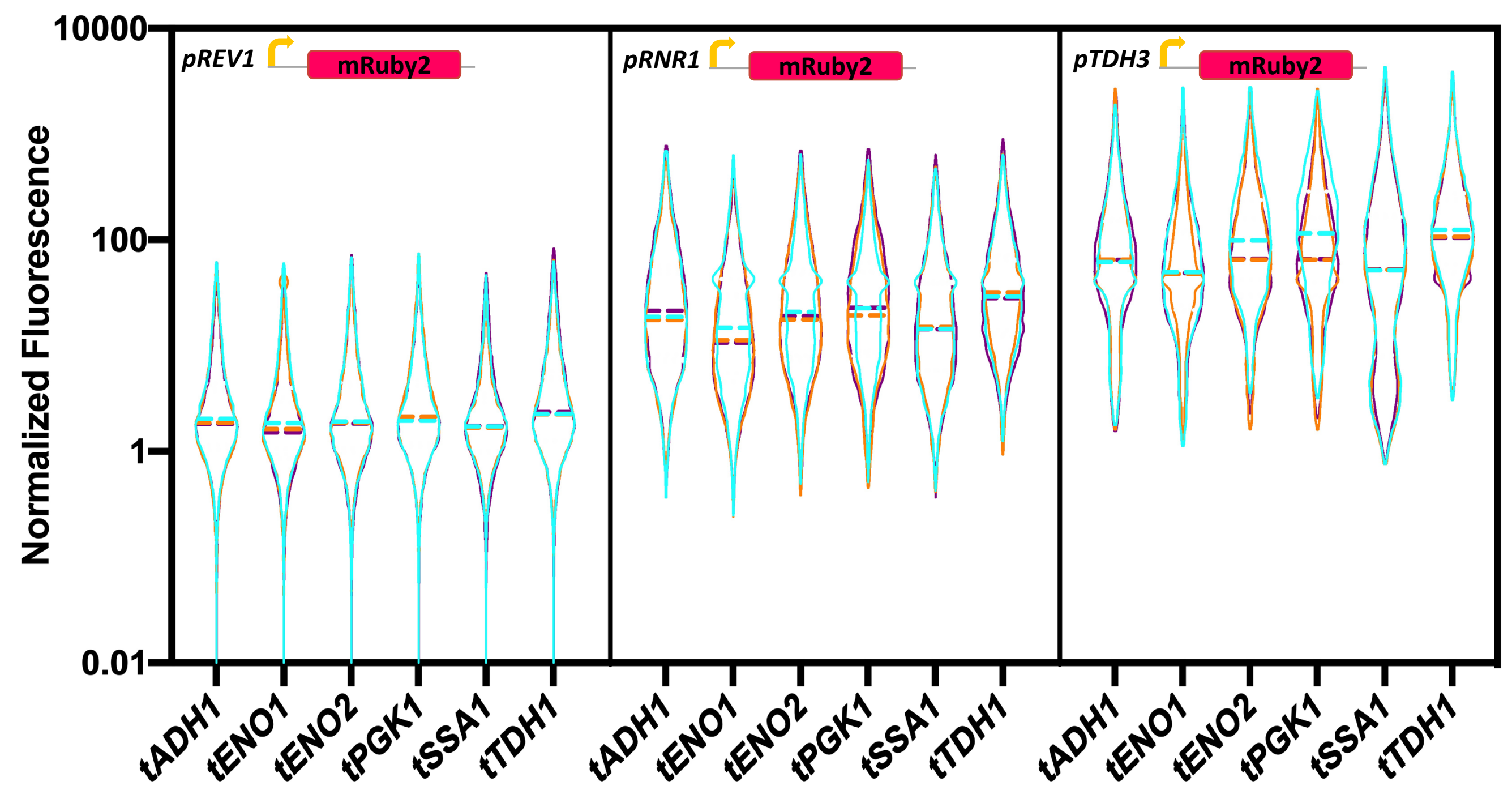

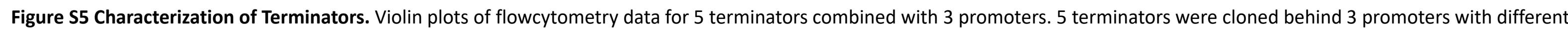

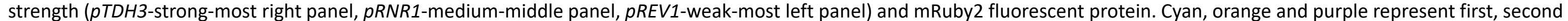
and third biological replicates, respectively. Each flowcytometry run consists of 10000 events. 


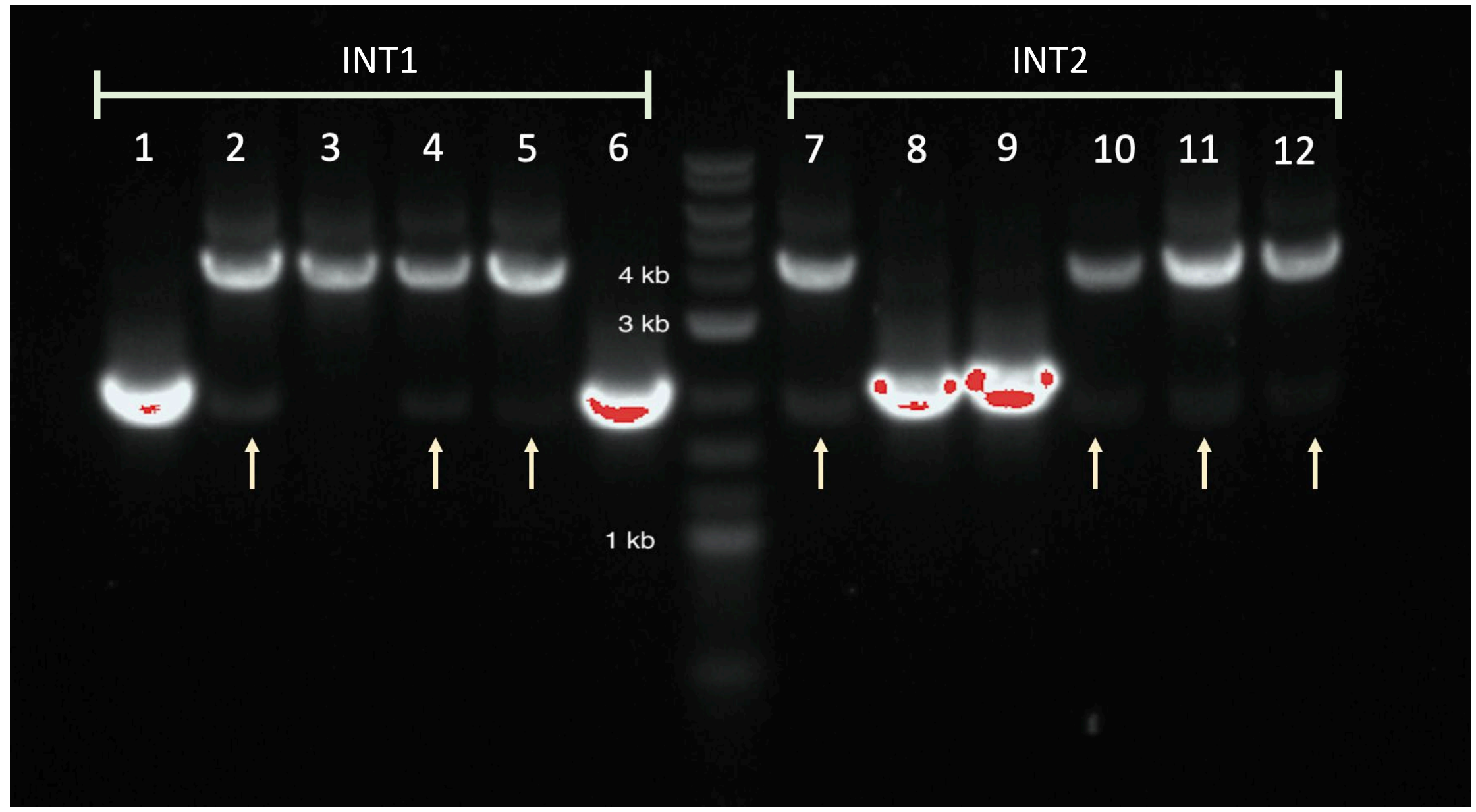

Figure S6. Colony PCR of $S$. boulardii genome editing using only homologous recombination. The gel image shows that when only donor DNA is transformed into $S b$, the cells keeps a copy of the original chromosomal locus sequence. Lanes 1-6 lanes show a PCR for testing integration into INT1. Lanes 7-12 show a PCR for testing integration into INT2. In both cases, a correct integration will yield a PCR product of $3.8 \mathrm{~kb}$, while the native sequence yields a PCR product of $2.0 \mathrm{~kb}$. 
a

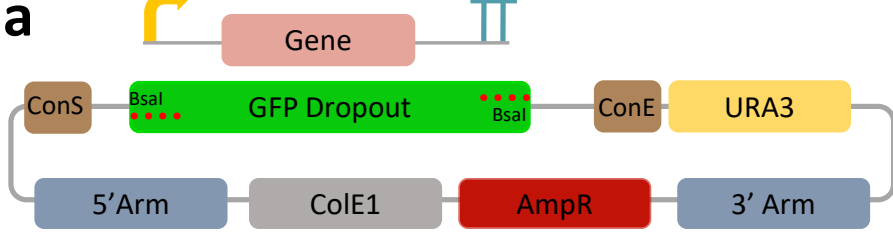

b

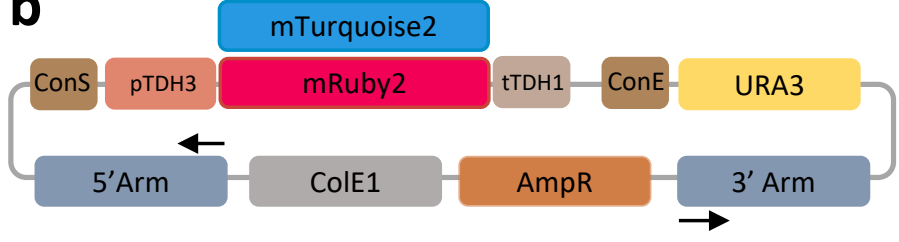

C

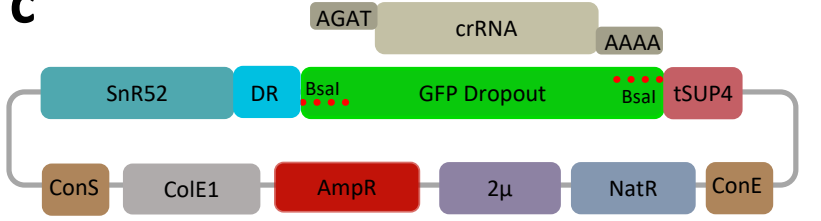

d

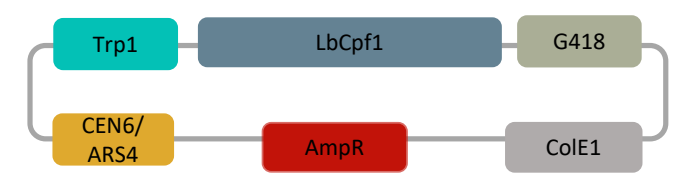

e
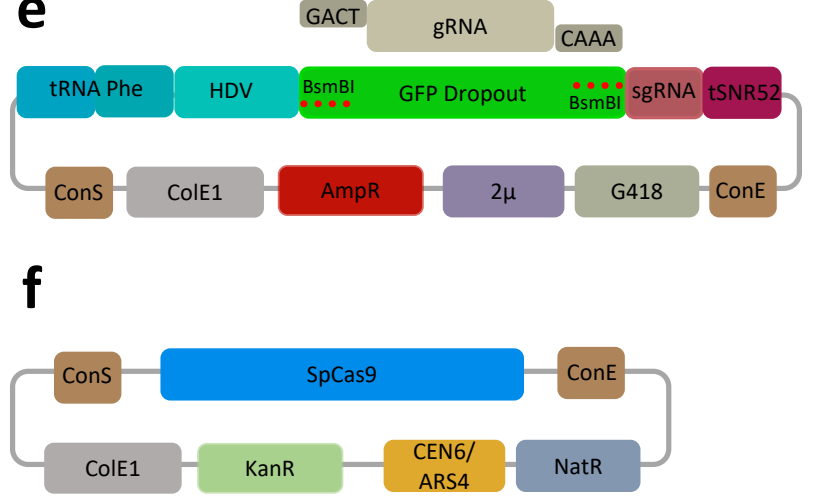

Figure S7. Genome editing plasmids. a) Vector for assembling the promoter, gene and terminator of interest. b) Example of constructed integration plasmid using cloning vector from a. c) Cloning vector with GFP dropout for constructing the CRISPR/LbCas12a crRNA. d) CRISPR/LbCas12a expression vector. e) Cloning vector with GFP dropout for constructing the CRISPR/SpCas9 gRNA. f) CRISPR/SpCas9 expression vector. All GFP dropout vectors are used in Golden Gate reactions with either Bsal or BsmBl enzymes. 


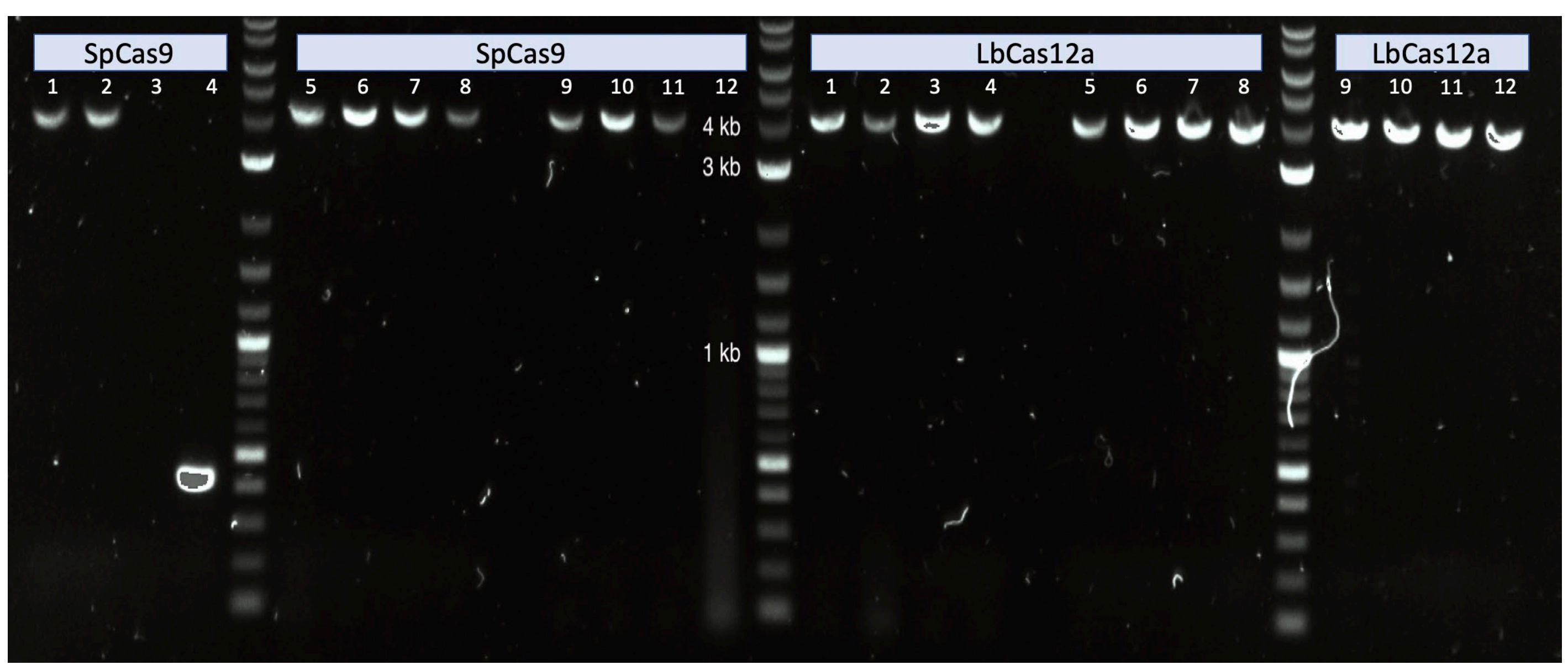

Figure S8. Colony PCR of $S$. boulardii genome editing using CRISPR/SpCas9 and CRISPR/LbCas12a. SpCas9 lanes 1-4 show a PCR screen for integration into INT1 (line 3 is failed PCR reaction and line 4 is unedited site), SpCas9 lanes 5-8 show a PCR screen for integration into INT2, and SpCas9 lanes 9-12 show a PCR screen for integration into INT3. LbCas12a lanes 1-4 show a PCR screen for integration into INT1, LbCas12a lanes 5-8 show a PCR screen for integration into INT2 and LbCas12a lanes 9-12 show a PCR screen for integration into INT3. 


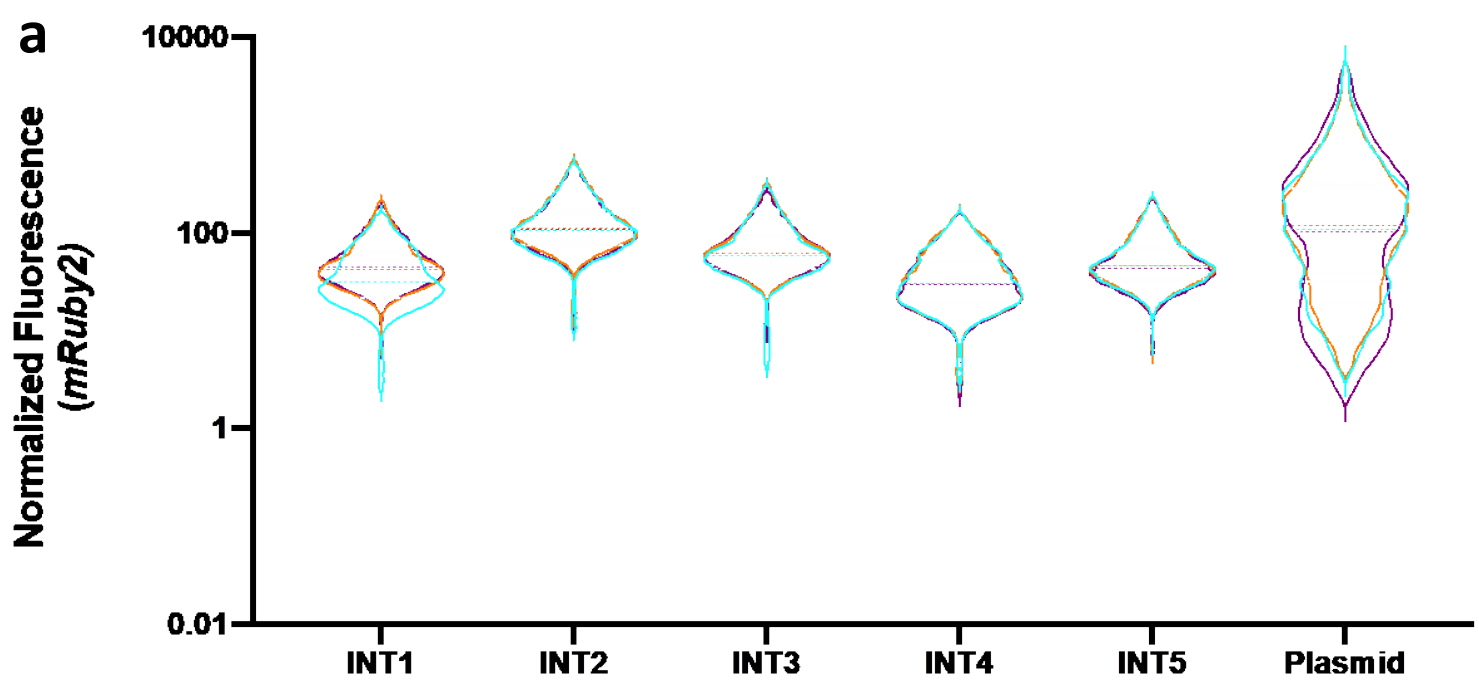

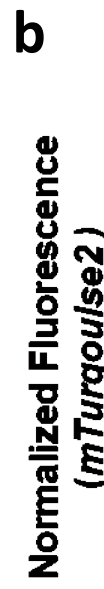

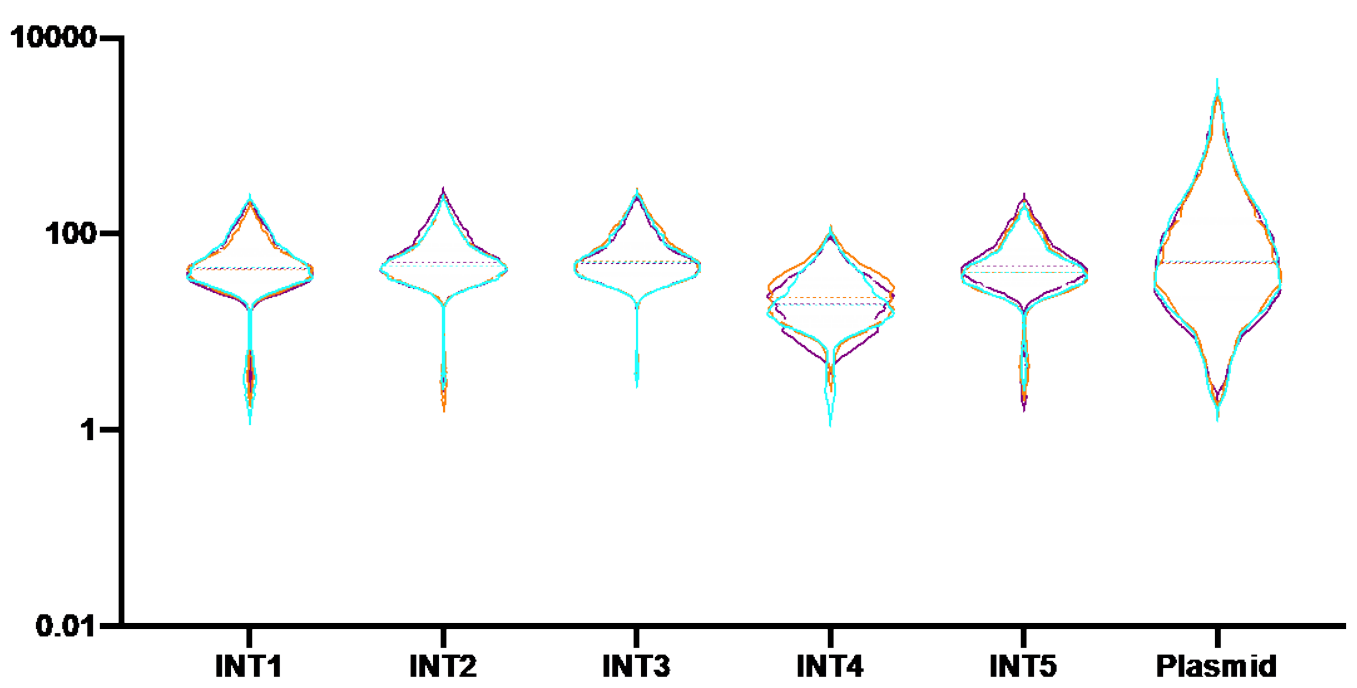

C

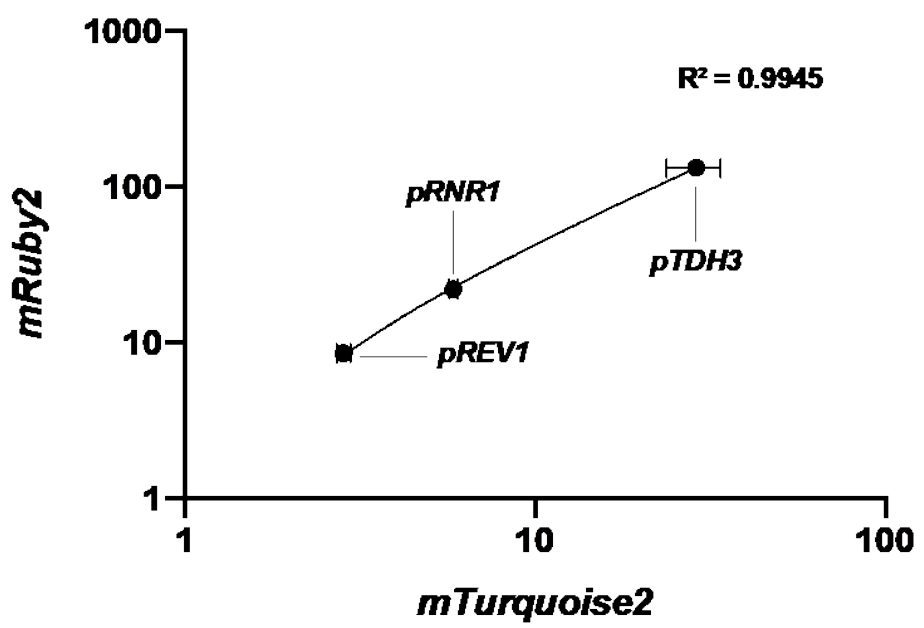

Figure S9. Effect of chromosomal locus and promoter choice on the expression of the fluorescent genes $m$ Ruby 2 and $m$ Turqouise2. Violin plots of flow cytometry data for a) $m$ Ruby 2 expression in 5 different loci in the genome compared to the expression of the same construct in plasmid with $2 \mu$. b) $m$ Turqiouse 2 expression in 5 different loci in the genome compared to the expression of the same construct in plasmid with $2 \mu$ origin. $c$ ) Comparison of constitutive promoter activity expressing mRuby2 ( $y$-axis) and mTurquoise2 ( $x$-axis). pREV, pRNR1 and pTDH3 promoters were cloned upstream of either mRuby2 or mTurquoise2 gene, integrated in the same locus (INT2), and plotted to determine the correlation in expression for the same promoters expressing different genes $\left(R^{2}=0.9945\right)$. 
a

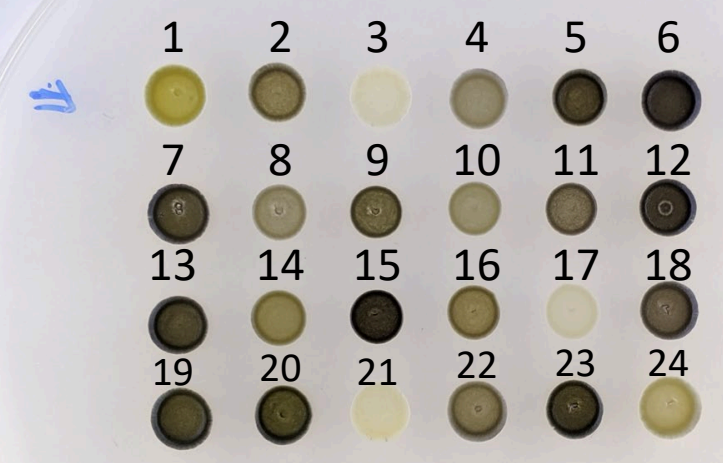

Figure S10. in vivo Combinatorial Pathway Assembly in S. boulardii. Sb Transformation plates for violacein (a) and $\beta$-carotene (b) pathways. Violacein (c) and $\beta$ carotene $(\mathbf{d})$ isolates picked from plates $a \& b$.

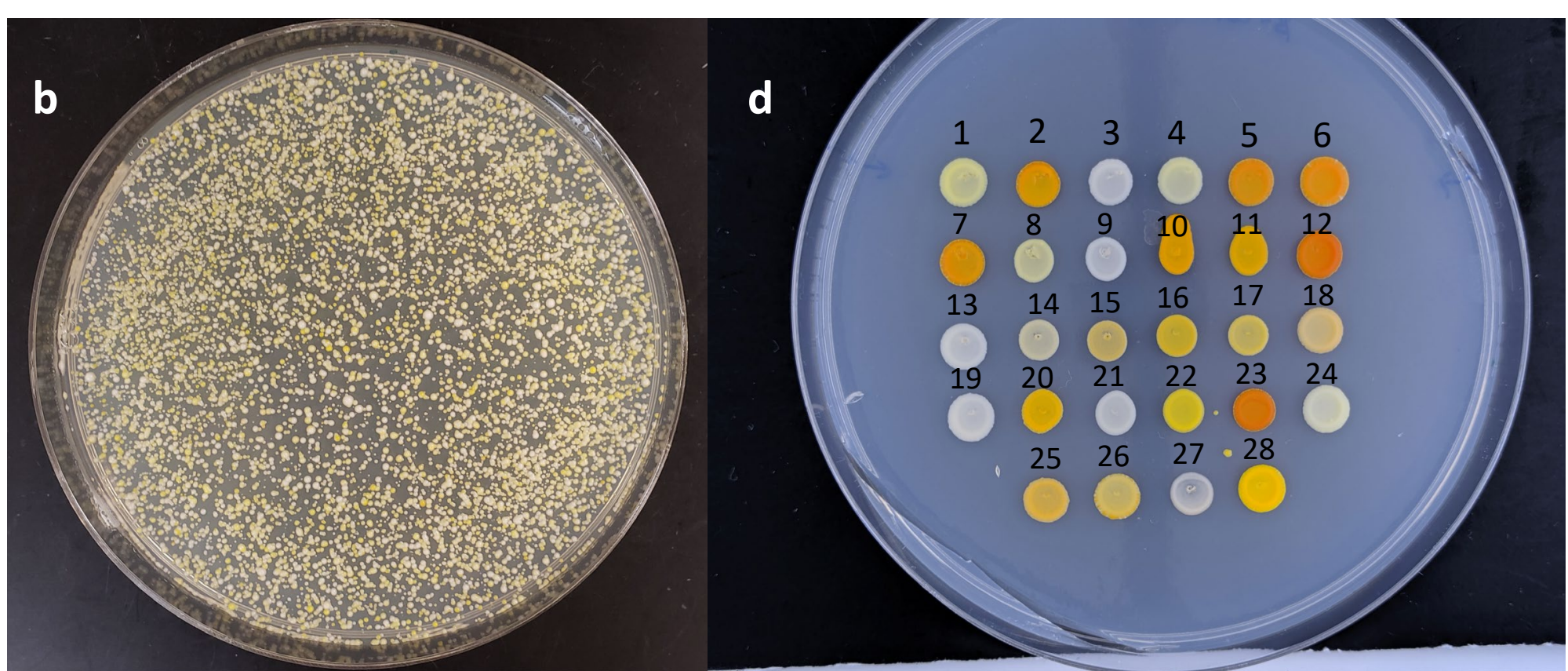




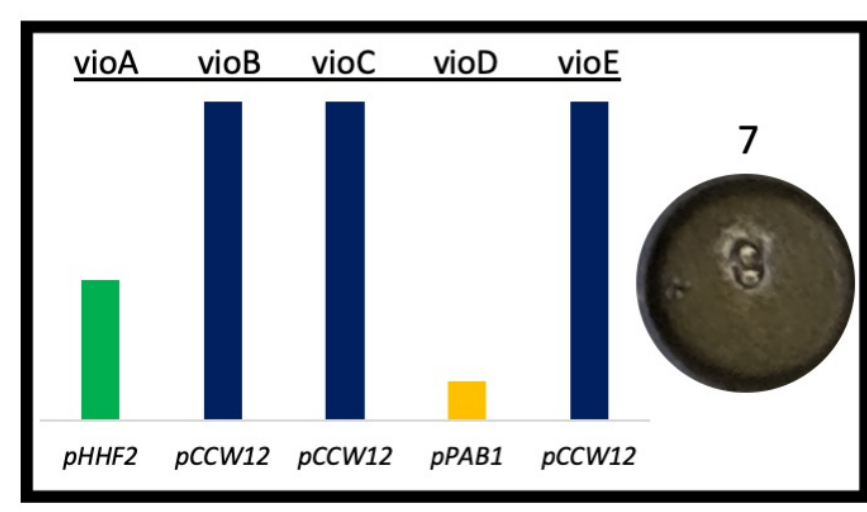

PPAB1 PHHF2

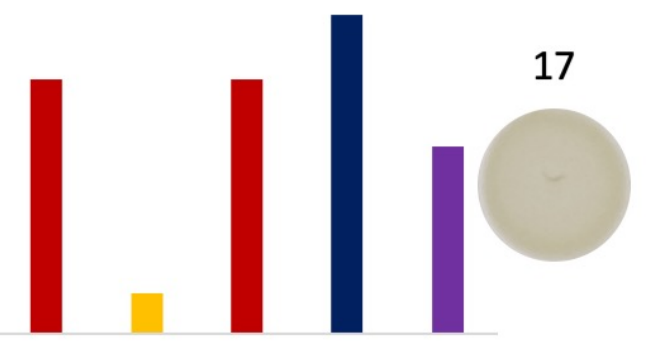

PTDH3

14

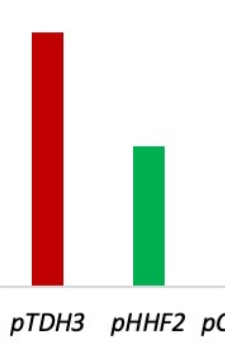

$$
\text { p }
$$

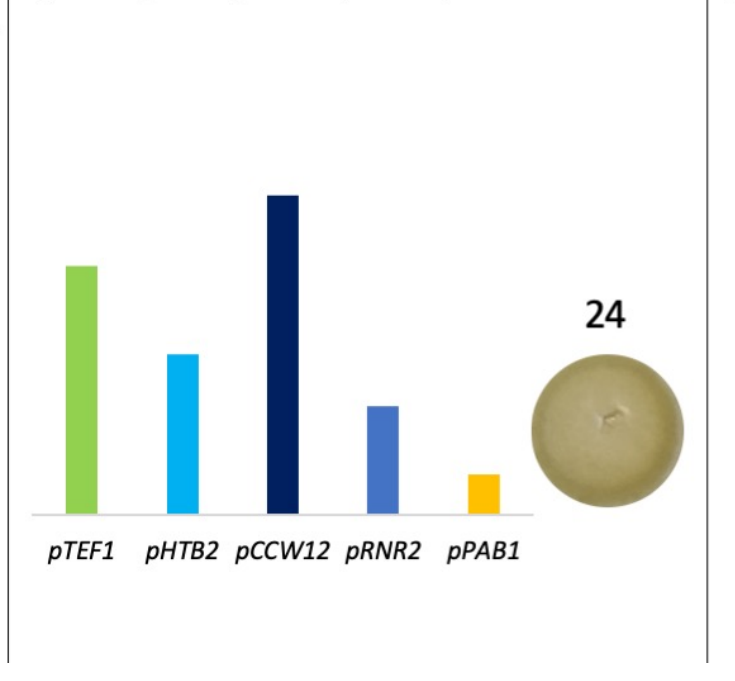

рTDH3 рPAB1

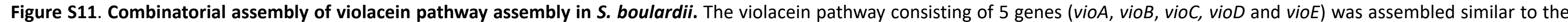

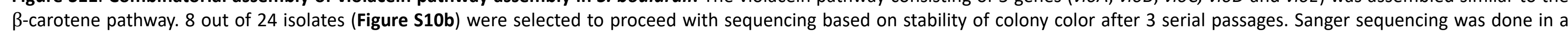

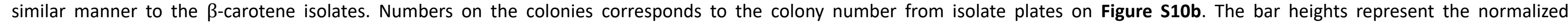
fluorescence values in linear scale for each promoter according to promoter characterization work (Figure 1a). 


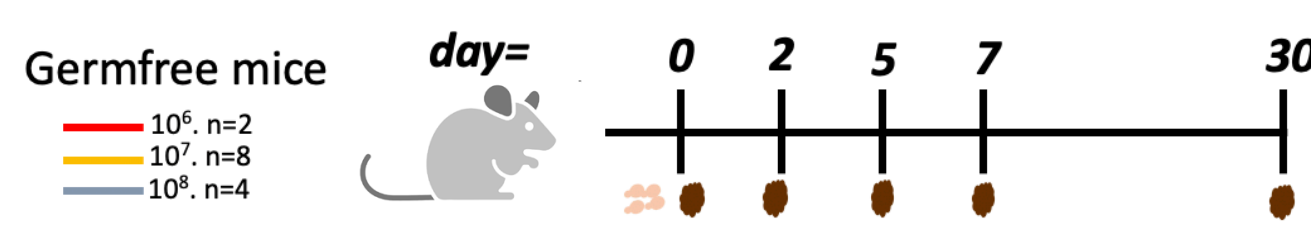

b

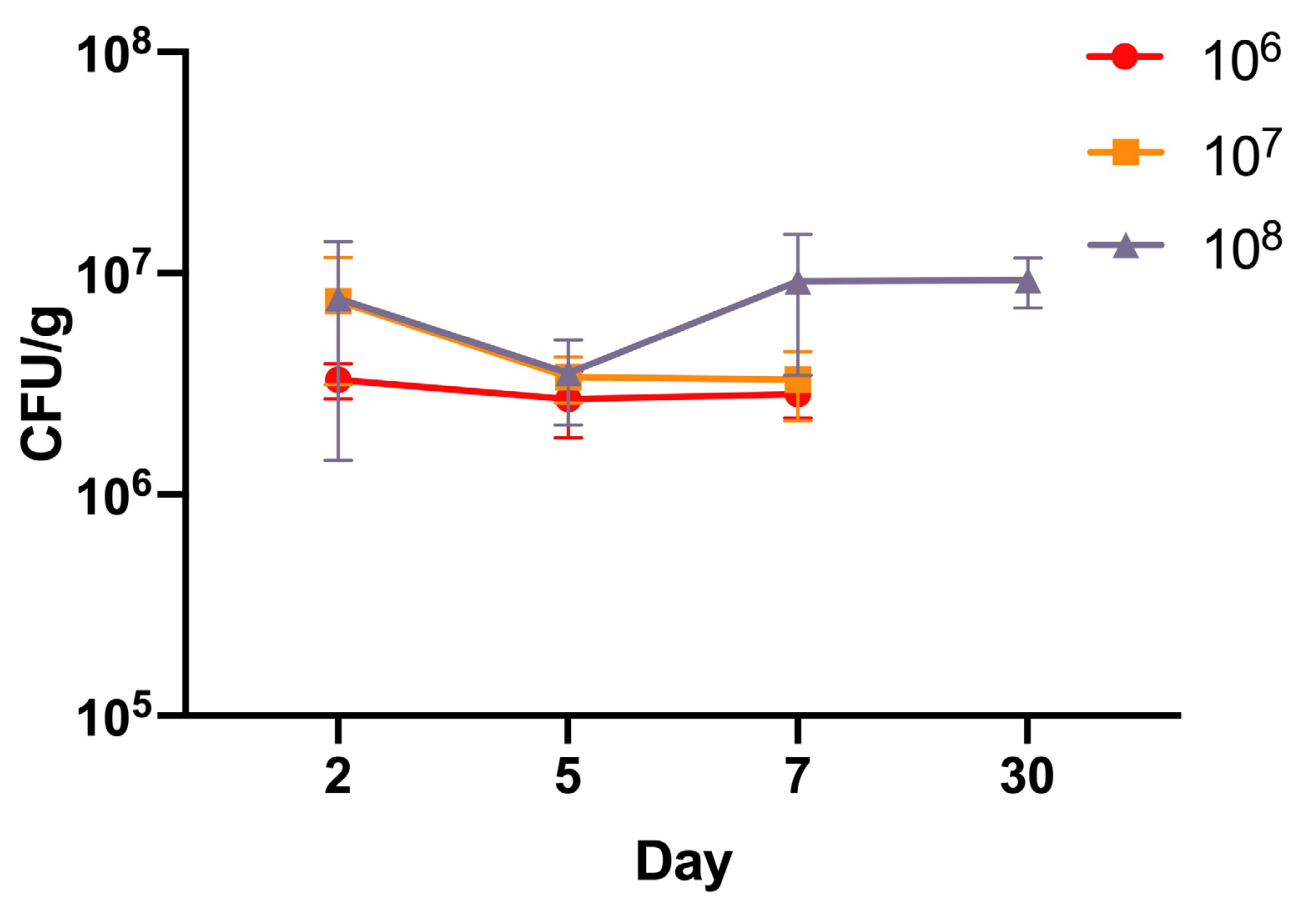

Figure S12. Dosage impacts the residence time of $S$. boulardii in germfree mice. a. Germfree mice were gavaged with 3 different amounts of $S b\left(10^{6}, 10^{7}\right.$ and $10^{8}$ cells) on day 0 . b. Residence time of $S b$ in germfree mice. Fecal samples were collected as shown in the timeline. Error bars indicate the standard deviation observed among 2 germfree mice for $10^{6} \mathrm{CFU}$ treatment, 8 germfree mice for $10^{7} \mathrm{CFU}$ treatment, and 4 germfree mice for $10^{8} \mathrm{CFU}$ treatment In all mouse models. Feces were collected prior to $\mathrm{Sb}$ gavage during days when both occurred. 

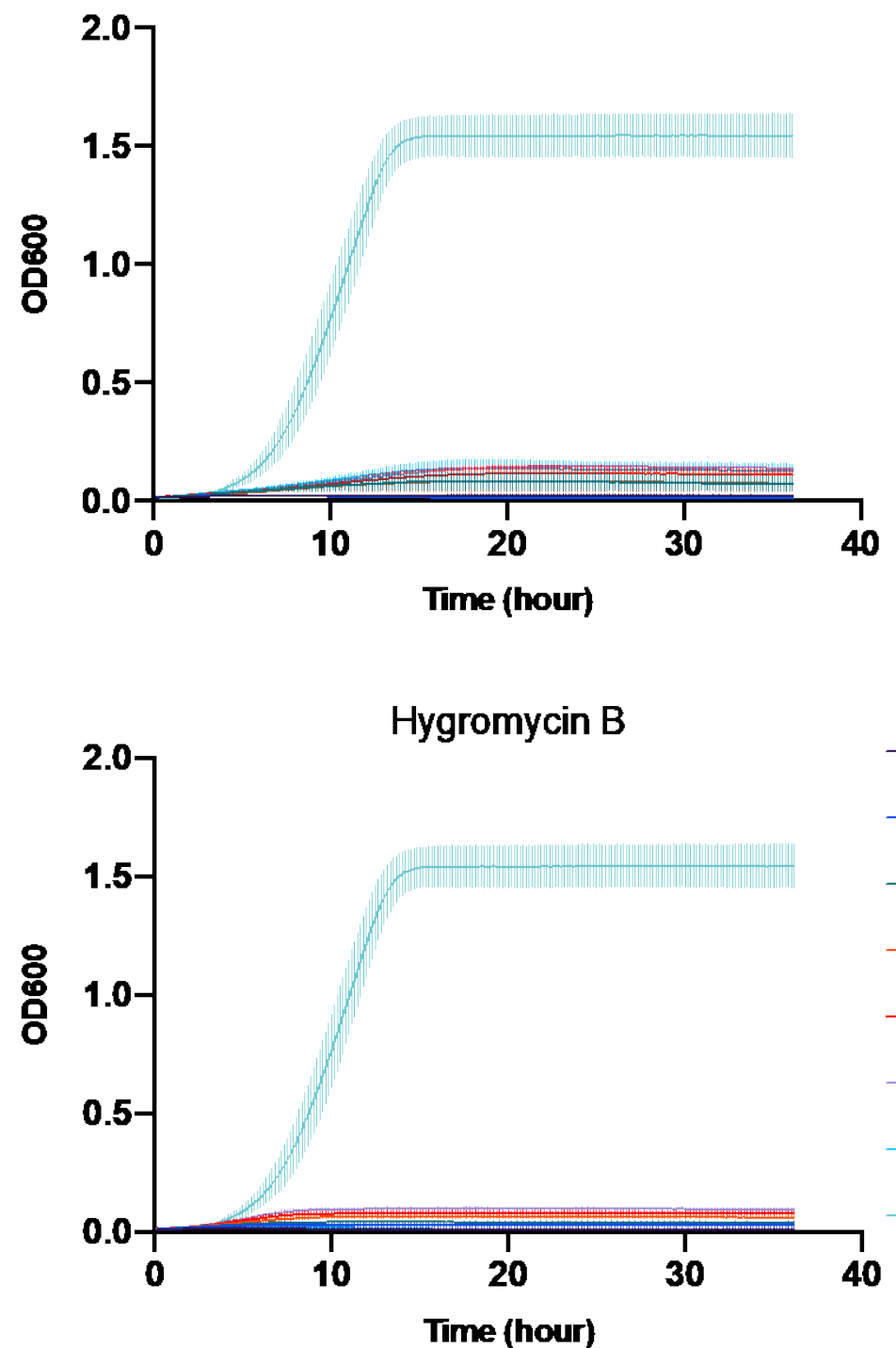
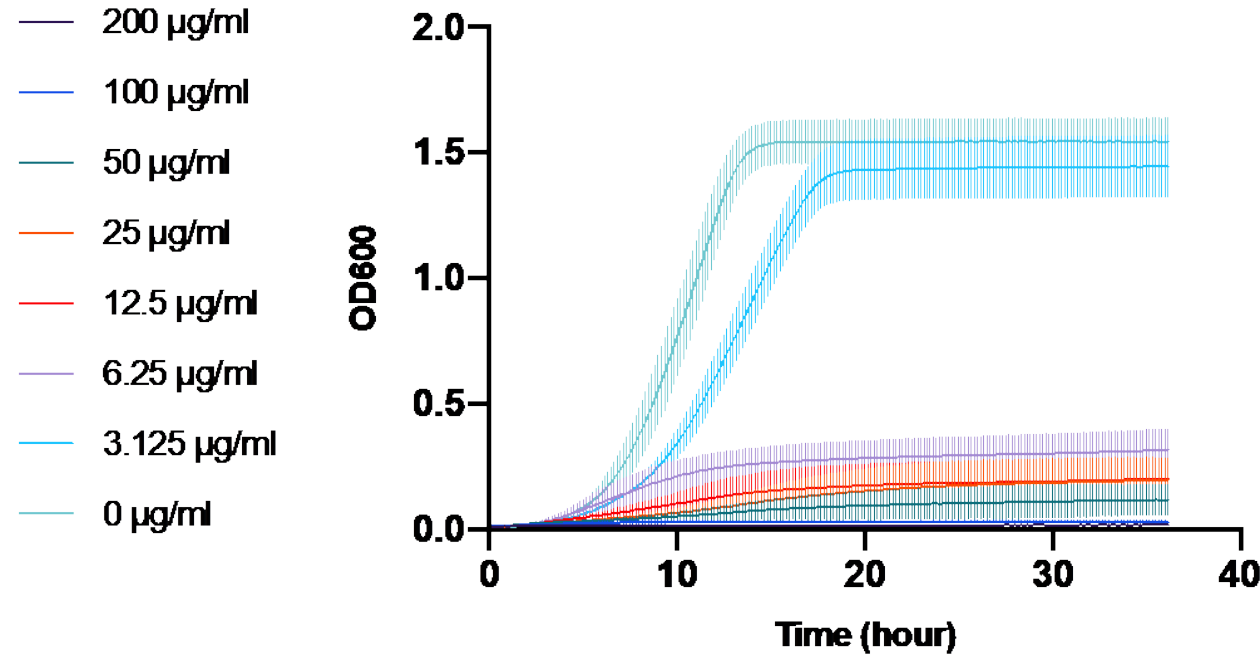

$-200 \mu g / \mathrm{ml}$

$-100 \mu \mathrm{g} / \mathrm{ml}$

$-50 \mu \mathrm{g} / \mathrm{ml}$

$25 \mu \mathrm{g} / \mathrm{ml}$

$-12.5 \mu \mathrm{g} / \mathrm{ml}$

- $6.25 \mu \mathrm{g} / \mathrm{ml}$

$3.125 \mu \mathrm{g} / \mathrm{m}$

$0 \mu \mathrm{g} / \mathrm{ml}$

- $200 \mu \mathrm{g} / \mathrm{ml}$

$-100 \mu \mathrm{g} / \mathrm{ml}$

- $50 \mu \mathrm{g} / \mathrm{ml}$

$25 \mu \mathrm{g} / \mathrm{ml}$

- $12.5 \mu \mathrm{g} / \mathrm{ml}$

$6.25 \mu \mathrm{g} / \mathrm{ml}$

$3.125 \mu \mathrm{g} / \mathrm{m}$ $0 \mu \mathrm{g} / \mathrm{m}$

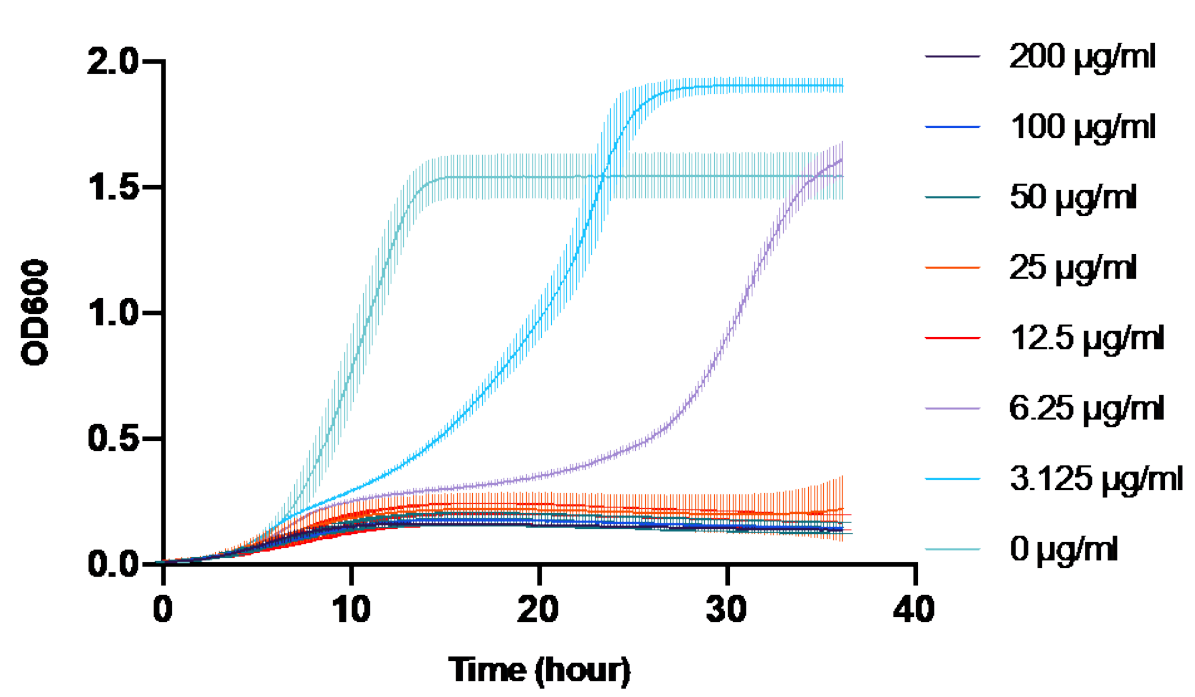

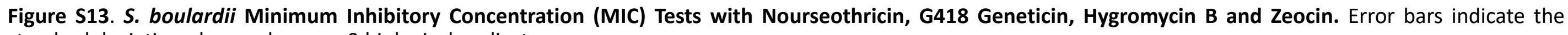
standard deviation observed among 3 biological replicates. 


\begin{tabular}{|c|c|c|c|c|c|}
\hline MoClo kit & Promoter & Score & Expect & Identities & Gaps \\
\hline YTK009 & $p T D H 3$ & 1218 bits(659) & 0 & $673 / 680(99 \%)$ & $0 / 680(0 \%)$ \\
\hline YTK010 & $p C C W 12$ & 1234 bits $(668)$ & 0 & $690 / 700(98 \%)$ & $4 / 700(0 \%)$ \\
\hline YTK011 & $p P G K 1$ & 1282 bits(694) & 0 & $698 / 700(99 \%)$ & $0 / 700(0 \%)$ \\
\hline YTK012 & $p H H F 2$ & 1197 bits(648) & 0 & $667 / 676(99 \%)$ & $1 / 676(0 \%)$ \\
\hline YTK013 & $p T E F 1$ & 1271 bits(688) & 0 & $696 / 700(99 \%)$ & $0 / 700(0 \%)$ \\
\hline YTK014 & $p T E F 2$ & 917 bits(496) & 0 & $534 / 550(97 \%)$ & $11 / 550(2 \%)$ \\
\hline YTK015 & $p H H F 1$ & 1254 bits(679) & 0 & $694 / 701(99 \%)$ & $1 / 701(0 \%)$ \\
\hline YTK016 & $p H T B 2$ & 1210 bits(655) & 0 & $683 / 696(98 \%)$ & $4 / 696(0 \%)$ \\
\hline YTK017 & $p R P L 18 B$ & 1236 bits(669) & 0 & $690 / 700(99 \%)$ & $2 / 700(0 \%)$ \\
\hline YTK018 & $p A L D 6$ & 1266 bits(685) & 0 & $695 / 700(99 \%)$ & $0 / 700(0 \%)$ \\
\hline YTK019 & $p P A B 1$ & 1271 bits(688) & 0 & $697 / 701(99 \%)$ & $1 / 701(0 \%)$ \\
\hline YTK020 & $p R E T 2$ & 1288 bits(697) & 0 & $699 / 700(99 \%)$ & $0 / 700(0 \%)$ \\
\hline YTK021 & $p R N R 1$ & 1266 bits(685) & 0 & $695 / 700(99 \%)$ & $0 / 700(0 \%)$ \\
\hline YTK022 & $p S A C 6$ & 1266 bits(685) & 0 & $695 / 700(99 \%)$ & $0 / 700(0 \%)$ \\
\hline YTK023 & $p R N R 2$ & 1251 bits(677) & 0 & $695 / 703(99 \%)$ & $4 / 703(0 \%)$ \\
\hline YTK024 & $p P O P 6$ & 1168 bits(632) & 0 & $688 / 712(97 \%)$ & $15 / 712(2 \%)$ \\
\hline YTK025 & $p R A D 27$ & 1184 bits(641) & 0 & $678 / 694(98 \%)$ & $9 / 694(1 \%)$ \\
\hline YTK026 & $p P S P 2$ & 1236 bits(669) & 0 & $689 / 699(99 \%)$ & $0 / 699(0 \%)$ \\
\hline YTK027 & $p R E V 1$ & 1251 bits(677) & 0 & $693 / 700(99 \%)$ & $4 / 700(0 \%)$ \\
\hline YTK028 & $p M F A 1$ & 1271 bits(688) & 0 & $697 / 701(99 \%)$ & $1 / 701(0 \%)$ \\
\hline
\end{tabular}




\begin{tabular}{|l|c|c|}
\hline \multicolumn{1}{|c|}{ Site } & Chromosome & Locus \\
\hline INT 1 & $\mathbf{V}$ & YERC 88 \\
\hline INT 2 & $\mathbf{X I}$ & non-coding region between SRP40 (YKR092C) and PTR2 (YKR093W) \\
\hline INT 3 & $\mathbf{X V I}$ & YPRCT3 \\
\hline INT 4 & $\mathbf{V I I I}$ & YHRC 14 \\
\hline INT 5 & $\mathbf{X V}$ & non-coding region between NTR1(YOR071c) and GYP1 (YOR070c) \\
\hline
\end{tabular}

Table S2. Integration locations (INT1-INT5) in S. boulardii's genome. 


\begin{tabular}{|c|l|l|}
\hline INT site & \multicolumn{1}{|c|}{ SpCas9 guide } & \multicolumn{1}{c|}{ LbCpf1 guide } \\
\hline \multirow{3}{*}{1} & ACTCCTGGGAGAGAACATTC & TCTTCCTTCCCGTTATACTTTGT \\
\cline { 2 - 3 } & AACTAGTATACCATCATATA & ATAATTGTTCGGATTGCATTGTT \\
\cline { 2 - 3 } & AATTCACAAAAGAGAATCGA & TGAATTCCTATAACGTCGGGAGA \\
\hline \multirow{3}{*}{2} & TTTCAACGCTTTCGAAGATG & TGTCAGATGTCATTATGGTCTCC \\
\cline { 2 - 3 } & GAATACTCAGACGTTTTCTG & CAGCCACATCTACAGGATGTTGA \\
\cline { 2 - 3 } & CAGAACCGTGACGCAATCTG & CCGGAAAGCTAAGAGTTTTCTG \\
\hline \multirow{3}{*}{3} & GGCTAATATAATGGTGATTC & GCAACCCAAGACTCGGCATACCA \\
\cline { 2 - 3 } & GATCAATATAACAATGCGTC & ACGTTTCCTTTGCAATAGGTAC \\
\cline { 2 - 3 } & ACAGTGCTGAAGCAGAGATA & GAATATATCTCTCCAATACAGCG \\
\hline 4 & ACAAATGTCAGAAATATGGC & TCATACAACCAATGATTAATGTC \\
\hline 5 & TATTAGAACCAGGGAGGTCC & AAAGATCCATACTTCTCTACATT \\
\hline
\end{tabular}

Table S3. Guide RNA sequences used for genome editing assisted with the CRISPR nucleases SpCas9 or LbCas12a. 
a

\begin{tabular}{|c|c|c|c|}
\hline & \multicolumn{3}{|c|}{ Genome Editing Method } \\
\hline Site & SpCas9 & LbCas12a & DNA \\
\hline \multirow{3}{*}{ INT 1 } & $191 / 232$ & $399 / 424$ & $34 / 72$ \\
\cline { 2 - 4 } & $531 / 603$ & $186 / 199$ & $44 / 68$ \\
\cline { 2 - 4 } & $237 / 275$ & $397 / 401$ & $21 / 58$ \\
\hline \multirow{4}{*}{ INT 2 } & $526 / 627$ & $751 / 795$ & $54 / 98$ \\
\cline { 2 - 4 } & $588 / 679$ & $743 / 784$ & $62 / 81$ \\
\cline { 2 - 4 } & $299 / 358$ & $782 / 839$ & $46 / 73$ \\
\hline \multirow{4}{*}{ INT 3 } & $503 / 597$ & $679 / 716$ & $19 / 92$ \\
\cline { 2 - 4 } & $280 / 328$ & $632 / 662$ & $41 / 142$ \\
\cline { 2 - 4 } & $242 / 294$ & $729 / 764$ & $38 / 107$ \\
\hline
\end{tabular}

b

\begin{tabular}{|c|c|c|c|}
\hline & \multicolumn{3}{|c|}{ Genome Editing Efficiency \% } \\
\hline Site & SpCas9 & LbCas12a & DNA \\
\hline \multirow{3}{*}{ INT 1 } & 82.3 & 94.1 & 47.2 \\
\cline { 2 - 4 } & 88.1 & 93.5 & 64.7 \\
\cline { 2 - 4 } & 86.2 & 99.0 & 36.2 \\
\hline Avg INT 1 & 85.5 & 95.5 & 49.4 \\
\hline \multirow{3}{*}{ INT 2 } & 83.9 & 94.5 & 55.1 \\
\cline { 2 - 4 } & 86.6 & 94.8 & 76.5 \\
\cline { 2 - 4 } & 83.5 & 93.2 & 63.0 \\
\hline \multirow{3}{*}{ Avg INT 2 } & 84.7 & 94.1 & 64.9 \\
\hline \multirow{2}{*}{ INT 2 } & 84.3 & 94.8 & 20.7 \\
\cline { 2 - 4 } & 85.4 & 95.5 & 28.9 \\
\cline { 2 - 4 } & 82.3 & 95.4 & 35.5 \\
\hline Avg INT 3 & 84.0 & 95.2 & 28.3 \\
\hline
\end{tabular}

Table S4. S. boulardii genome editing efficiencies using 3 different methods. a. Number of colonies screened for each editing method. Successfully edited cells are defined by the number of fluorescent (mTurquoise2) colonies divided by the total number of colonies on the plate $\mathbf{b}$. The percentage of successfully edited cells in the 3 different locations using 3 different editing methods. 


\section{Supplementary Text}

\section{Beta-carotene production rate calculations}

1. Day 0 (pre-gavage) $\beta$-carotene ( $\mathrm{mg} / \mathrm{g}$ feces) values for each mouse ( 7 mice in total) were subtracted from Day 2-Day 14 (post-gavage) values of $\beta$-carotene ( $\mathrm{mg} / \mathrm{g}$ feces) to compensate for baseline $\beta$-carotene levels. Then, these values were divided by the $S$. boulardii load (CFU/g feces) to obtain daily values of $\beta$-carotene $(\mathrm{mg}$ ) per viable $S$. boulardii cells Finally, these values were averaged across 7 mice and 5 sample collection points to obtain average $\beta$-carotene $(\mathrm{mg})$ per viable $S$. boulardii cell.

2. In order to calculate overall titer of $\beta$-carotene synthesized in situ, we applied Production $_{\beta \text {-carotene }}=$ Out $_{\beta \text {-carotene }}-I_{\beta}$-carotene , where Out $_{\beta \text {-carotene }}$ corresponds to average total $\beta$-carotene $(\mathrm{mg})$ obtained in feces throughout the experiment and $I n_{\beta}$-carotene corresponds to $\beta$-carotene $(\mathrm{mg})$ gavaged to the mice on Day 0 . Out $t_{\beta \text {-carotene }}$ was calculated by (i) taking the average of Day 2-Day 14 (post-gavage, Day 0 subtracted) values of $\beta$-carotene across 7 mice, which was found to be $14.14 \mu \mathrm{g} / \mathrm{g}$ feces, and multiplying it by (ii) daily feces output $(1 \mathrm{~g})$ and (iii) duration of the experiment (14 days) which yielded an output of $197.9 \mu \mathrm{g} \beta$-carotene per mouse. $\operatorname{In}_{\beta}$-carotene was calculated as $3.5 \mu \mathrm{g}$, based on the per-cell in vitro $\beta$-carotene measurements and the number of gavaged cellls. By applying the equation, the overall $\beta$-carotene titer synthesized in situ was found to be $194.4 \mu \mathrm{g}$. 